\title{
An Experimental Study on Heat Transfer Enhancement of Flat Plates using Dimples
}

\author{
Neha G. Katarwar* and R.K.Patil ${ }^{\ddagger}$ \\ ${ }^{\dagger}$ Mechanical Engineering, Savitribai Phule Pune University Pune, Maharashtra, India
}

Accepted 15 June 2016, Available online 20 June 2016, Special Issue-5 (June 2016)

\begin{abstract}
Dimples play significant role in heat transfer enhancement of electronic components cooling systems. Heat transfer describes the exchange of thermodynamic energy, between actual systems depending on the temperature and pressure, by dissipating heat. The basic modes of heat transfer are conduction or diffusion, convection and radiation. Heat flow always occurs from a region of high temperature to another region of lower temperature. Heat transfer changes the internal energy of the given systems involved according to the First Law of Thermodynamics. In case of natural convection is a mechanism, or type of heat flow, in which the fluid flow is not generated by any external source (like a suction device, fan, pump, etc.) but only by density differences in the fluid occurring due to temperature variation. In natural convection, fluid surrounding a heat source absorbs heat, becomes less dense and rises. The surrounding, cooler fluid then moves to replace it. The performance of the system was carried out for Natural convection conditions with air as a working fluid. The overall heat transfer coefficient and Nusselt numbers at different sizes of dimples were obtained. From the obtained results, it was observed that the heat transfer coefficient and Nusselt numbers increases with increase in heat input to the system.
\end{abstract}

Keywords: Electronic Cooling, Natural Convection, Dimples, Passive Techniques

\section{Introduction}

Convective heat transfer is the transfer of heat from one place to another by the movement of fluids, this process is essential to the transfer of heat via mass transfer. Bulk motion of fluid enhances heat transport in many physical situations, such as between a solid surface and the fluid. Convection is usually the dominant form of heat transfer in liquids and gases. Although sometimes discussed in heat transfer, convection is usually used to describe the combined effects of heat conduction within the fluid and heat transference by bulk fluid flow streaming. Natural convection is a mechanism and type of heat transfer, in which the fluid motion is not generated by any external source like a pump, fan, suction device, etc. but it generated by density differences in the fluid occurring due to temperature gradients. In natural convection, fluid receives heat from the surrounding becomes less dense and rises. The surrounding, cooler fluid then moves to replace it. This cooler fluid is then heated and the process forming and convection current is continue. This process transfers heat energy from the bottom of the convection cell to top. The driving force for natural convection is buoyancy and result of differences in fluid density. Because of this, the

*Corresponding author: Neha G. Katarwar presence of a proper acceleration such as arises from resistance to gravity, or an equivalent force which is arising from acceleration, centrifugal force or Corollas effect is essential for natural Convection. For example, natural convection does not operate in free-fall inertial environments, such as that of the orbiting International Space Station, where other heat transfer mechanisms are required to prevent electronic components from overheating.

\section{Literature Review}

Many studies have focused on cooling through natural convective heat transfer in and many previous researchers investigated natural convection from the extended surfaces. These are motivated by the fact that the heat transfer rate from the extended surfaces differs greatly that from the base surfaces. Relevant literature pertaining to enhancement of heat transfer by introducing various methods mounted on the heat transfer surfaces reviewed from different points. A number of heat transfer studies from Russia utilize dimples. A variety of experimental, analytical and computational research works has been carried out on enhancement of heat transfer.

Kuethe et al. was the first suggest the use of dimple surface for heat transfer. Surface dimples are expected to promote turbulent mixing in the flow and enhance the heat transfer. 
M.A.Dafedar et al studied experimentally the heat transfer enhancement through various geometries of dimpled surfaces in longitudinal directions. In his paper horizontal rectangular plates of copper and aluminum with different dimpled geometries for inline arrangements were studied in natural convection with steady laminar external flow condition. The various parameters considered for study are Nusselt number, heat transfer coefficient and heat transfer rate for a constant Prandtl number (0.7) and Grashof number (104-107 ).It has been found that the heat transfer coefficient and heat transfer rate increases for various dimpled surfaces as compared to plane surface. It has been also found that the heat transfer coefficient and heat transfer rate increases with longitudinal direction as compared to lateral direction. And it is seen that heat transfer rate is maximum for triangular shape dimple when the apex of triangle is faced towards inlet of air flow. finally it is proved that heat transfer enlagerment takes place along the dimpled surface.

Iftikarahamad $\mathrm{H}$. Patel et al presented the computational analysis of convective heat transfer in turbulised flow past a dimpled surface. A parametric study is done with $\mathrm{k}-\varepsilon$ turbulence model to determine the effects of Reynolds number, dimple depth and Nusselt number on heat transfer enlargement. In this paper we have calculated heat transfer coefficients in a medium with one side dimpled surface. The sphere dimple geometry was considered with diameter (D) 10 $\mathrm{mm}$ and the depth $(\delta) 4 \mathrm{~mm}$, to obtain $\delta / \mathrm{D}$ ratio as 0.4 and it was increased later to $5 \mathrm{~mm}$ to increase $\delta / \mathrm{D}$ ratio to 0.5 . The Reynolds number based on the channel hydraulic diameter was varied from 200000 to 360000 .The conclusion is that more heat transfer was occurred ensuring of the dimples due to flow reattachment. Because of the flow recirculation on the upper side in the dimple, the heat transfer coefficient was very low. As the Reynolds number increased, the overall heat transfer coefficient was also increased.

Faheem Akthar et al experimental determination of the natural convection heat transfer over circular dimpled surfaces is carried out. The various heat transfer parameters mediated for study are Nusselt number, heat transfer coefficient and heat transfer rate. From the achieved results, it can be concluded that large amount of heat transfer enhancement does attain for the dimpled surfaces.

Saurabh R Verma et al studied Heat Transfer enhancement using dimples are depends on the principle of scrubbing action of cooling fluid taking place inside the dimple and phenomenon of built up the delay of flow separation over the surface. Spherical dimples have shown good heat transfer characteristics when used as surface roughness. The technology using dimples recently attracted interest due to the substantial heat transfer raised it induces, with pressure drop penalties smaller than with other types of heat augmentation. From all the research work studied the researchers have used various dimple shaped geometries such as triangular, ellipsoidal, circular, square out of which ellipsoidal shape gives better results because of to prior vortex formation then other shapes

Amjad Khan et al studied the fluid flow and heat transfer characteristics at different angle of orientation from the centre with apex facing the inlet were investigated. The experiment was carried out for laminar Natural convection conditions with air as a working fluid. The overall Nusselt numbers and heat transfer coefficient at different direction angle of dimples were obtained. From the obtained results, it was determine that the Nusselt numbers and heat transfer coefficient increases with decrease in the bearing angle of dimples.

Burgess et al. experimentally analyzed the effect of dimple depth on the surface within a channel with the ratio of dimple depth to dimple printed diameter, equal to $\delta / D, 0.1,0.2$, and 0.3 . The data showed that the local Nusselt number increased as the dimple depth increased due to an increased strength and intensity of vortices and three dimensional (3D) turbulent productions.

Ligrani et al. experimentally showed the influence of dimple aspect ratio, temperature ratio, Reynolds number and flow structures in a dimpled channel at $\mathrm{Re}$ $=600$ to 11,000 and air inlet stagnation temperature ratio of 0.78 to 0.94 with $\mathrm{H} / \mathrm{D}=0.20,0.25,0.5,1.00$. The results indicated that the vortex pairs which are systematically shed from the dimples become stronger and local Nusselt number increase as channel height decreases. As the temperature ratio Toi/Tw decreases, the local Nusselt number also increased.

Mahmood et al. studied the flow and heat transfer characteristics over staggered arrays of dimples with $\delta / D=0.2$. For the globally average Nusselt number, there were small changes with Reynolds number. He studied the effect of dimpled protrusions (bumps) on the opposite wall of the dimpled surface.

S. D. Hwang et al. in present study, heat transfer and thermal performance of a periodically dimple protrusion patterned surface have been investigated to enhance energy-efficiency in compact heat exchangers. The local heat transfer coefficients on the dimple/protrusion walls are derived using a transient TLC (Thermo chromic Liquid Crystal) technique at low Reynolds number. The periodically patterned surface is applied to the bottom wall only or both the bottom and top walls in the test duct. On the single-side patterned walls, various secondary flows generated from the dimple/protrusion coexist. The vortices induced from the upstream affect strongly on the downstream pattern. For the double-side patterned wall case, vortex interaction affected by the opposite wall enhances highly the heat transfer. The heat transfer augmentation is higher in the lower Reynolds number due to the efficient vortex interactions Therefore, the performance factor considering both heat transfer enhancement and pressure loss increases with decreasing the Reynolds number. 
Moon et al. shows that heat transfer is enlarged and pressure loss is abbreviated for dimpled surfaces. Some practical applications dimpled surfaces in internal passages include macro-and micro-scale heat exchangers, electronics cooling, combustion chamber liners, passages for internal cooling of turbine blades in gas turbine engines, biomedical devices, etc.

Sane et al. was carried out experiment to determine heat transfer and friction coefficient by dimpled surface with the aspect ratio of rectangular channel is kept 4:1 and Reynolds number based on hydraulic diameter is varied from 10000 to 40000 . They were observed that at all Reynolds number as depth increases from 0.2 to 0.3 , the number and thermal performance increases and then after when depth increase from 0.3 to 0.4 conducted Nusselt number and thermal performance decreases.

\section{Design and Development of Experimental Setup}

A schematic diagram of the experimental setup is as shown in Fig. 1. The experimental setup consists of the segment such as RTD's, Temperature Indicator, Heating element, and an arrangement to vary the heat input, Duct, Stand and Hanger etc.

\begin{tabular}{|lllll|}
\hline 0 & 0 & 0 & 0 & 0 \\
0 & 0 & 0 & 0 & 0 \\
0 & 0 & 0 & 0 & 0 \\
\hline
\end{tabular}
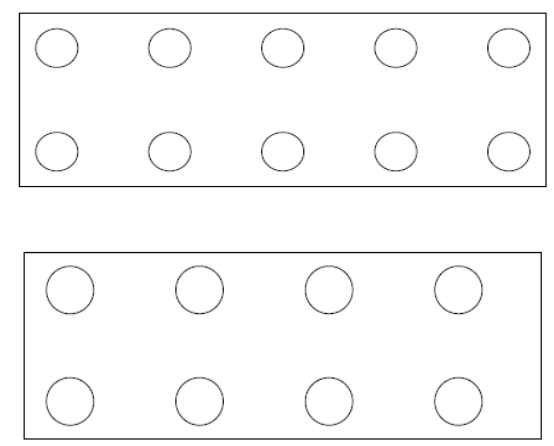

Fig.1 Dimpled plate with different diameter

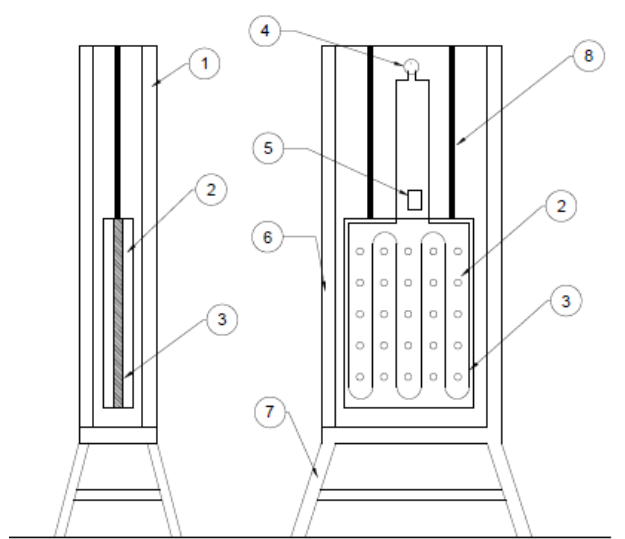

Fig 2. Schematic layout of experimental system
1- Cover

2- Dimple

3- Plate with dimples

4- Temperature Indicator

5- Voltmeter

6- Outer cover

7- Stand

8- Hanging wire

\section{Test Methodology}

In order to Experimental interpretation of Natural heat transfer coefficient through vertical heated roughened surface, it has been express to vary the heat input from $25 \mathrm{~W}$ to $100 \mathrm{~W}$ in the step of $25 \mathrm{~W}$. Readings are taken at steady state. Heater is placed between two test plates. Whole test set up is mounted vertically. Voltage supplied is varied with the help of dimerstat.

\subsection{Test Parameters and Calculations}

Experimentation was carried on the plane and three configuration of dimple on copper and aluminium plate

Table 3.1 Test parameters

\begin{tabular}{|c|c|}
\hline Parameter & Description \\
\hline Heat load (W) & 25W,50W,75W,100W \\
\hline Time & Up to Steady State \\
\hline
\end{tabular}

All the essencial components were assembled and experimental set was developed. The necessary instruments were attached at correct composition and the setup is ready for the experimentation.

\subsection{Formulae Used}

GrashofNumber(Gr)=

$$
\frac{g \times \beta \times \Delta \mathrm{T} \times \mathrm{L}^{3}}{\vartheta^{2}}
$$

Nusselt Number $(\boldsymbol{N u})=\mathrm{hL} / \mathrm{k}$

Relays Number $(\mathrm{Ra})=\mathrm{Gr} \times \mathrm{Pr}$

Heat Transfer coefficient $\left(\mathrm{h}_{\mathrm{th}}\right)=(N u) / k \mathrm{~L}$

\section{Results and Discussion}

Experiments were carried out by using four test plates one of these is plain plate and other three are dimple with different diameters. The effect of dimple on the heat transfer characteristics are presented by heat transfer coefficient and Nusselt number. The heat transfer coefficient and Nusselt number is calculated by changing Heat input to the dimple plate. The results are represent in Figs. 4.1-4.7. The deviations of various 
parameters are heat input, Nusselt Number and Heat transfer coefficient.

Fig 4.1 and 4.2 showed that the value of Nusselt Number calculated by experimental and theoretical method is same. From this graphs it is proved that experimental setup is justify.

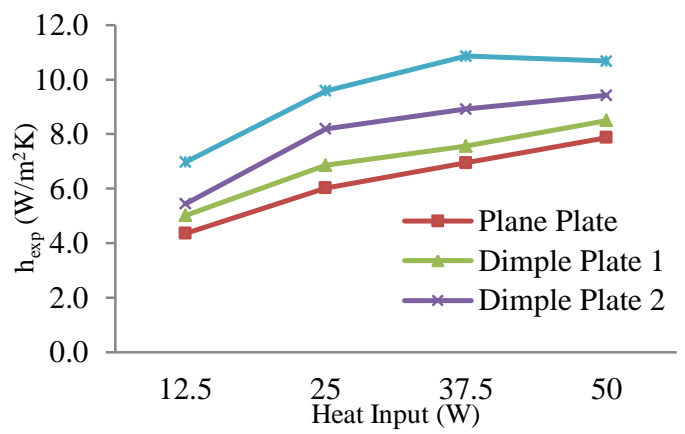

Fig 4.3: Variations in Heat Transfer Coefficient with Heat Input for various dimple Plates for copper plate.

For advancement in heat input, it can be seen from above graph that increase is found in experimental heat transfer coefficient in connection of dimple and plain plate. Above graph shows that the values of heat transfer coefficient are increases with increasing heat.

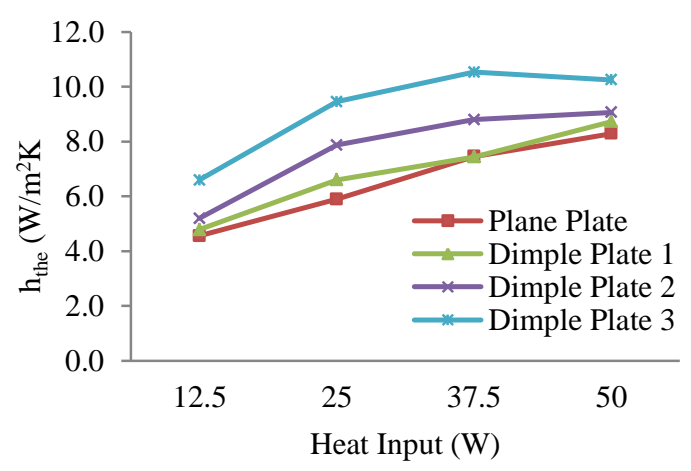

Fig 4.3: Variations in Heat Transfer Coefficient with Heat Input for various dimple Plates for copper plate.

For increment in heat input, it can be seen from above graph that enhancement is found in theoretical heat transfer coefficient in comparison of dimple and plain plate. Above graph shows that the values of heat transfer coefficient are increases with increasing heat input.

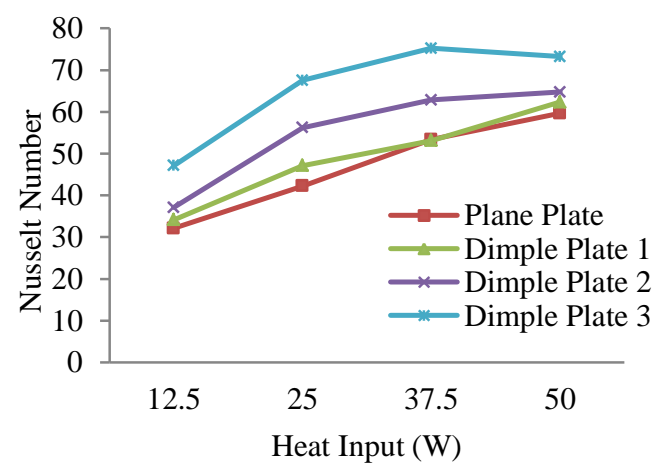

Fig 4.4: Variations in Nusselt Number with Heat Input for various dimples plate Plates for copper plate.
For increment in heat input, it can be seen from above graphs that enhancement is found in Nusselt Number in comparison of dimple and plain plate. Above figure shows that the values of Nusselt Number are increases with increasing heat input.

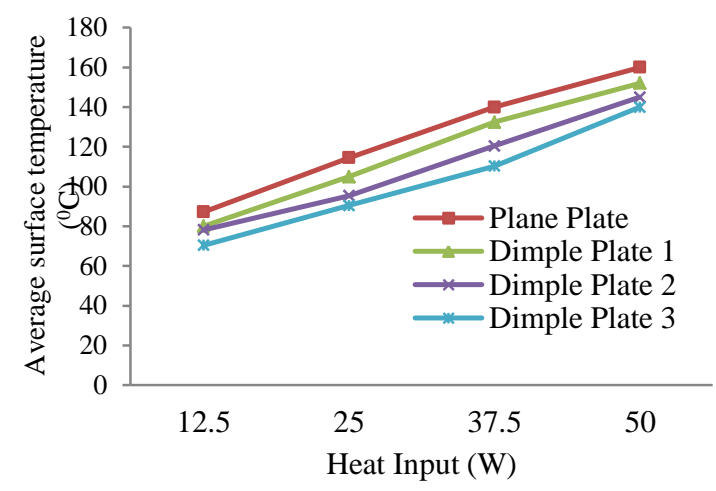

Fig 4.5: Variations in Surface temperature with Heat Input for various dimple plate for copper plate.

For increment in heat input, it can be seen from above graphs that enhancement is found in Surface Temperature in comparison of dimple and plain plate. Above figure shows that the values of Surface temperature are increases with increasing heat input.

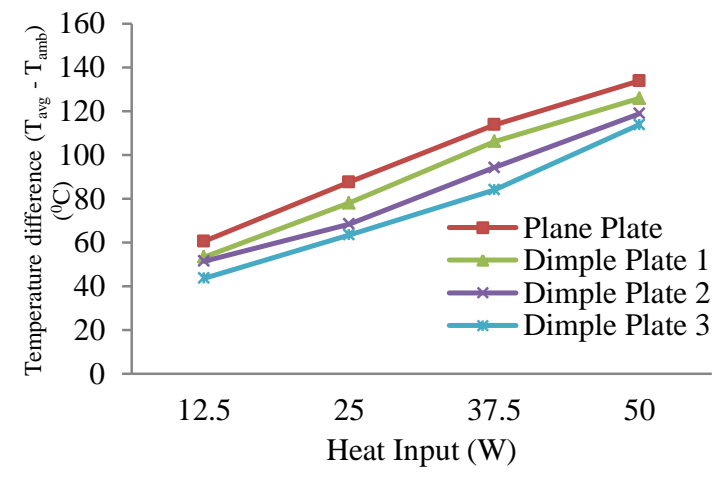

Fig 4.5: Variations in temperature difference with Heat Input for various dimple plate for copper plate.

For increment in heat input, it can be seen from above graphs that enhancement is found in temperature difference in comparison of dimple and plain plate. Above figure shows that the values of Surface temperature are increases with increasing heat input.

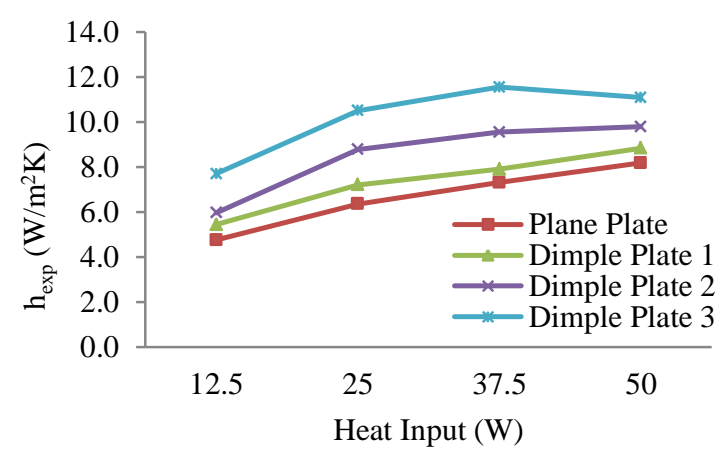

Fig 4.3: Variations in Heat Transfer Coefficient with Heat Input for various dimple Plates for Aluminum plate. 
For increment in heat input, it can be seen from above graph that enhancement is found in experimental heat transfer coefficient in comparison of dimple and plain plate. Above graph shows that the values of heat transfer coefficient are increases with increasing heat input.

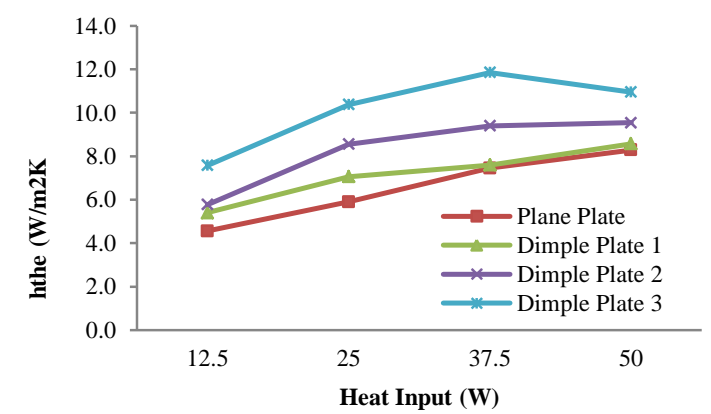

Fig 4.3: Variations in Heat Transfer Coefficient with Heat Input for various dimple Plates for Aluminum plate.

For increment in heat input, it can be seen from above graph that enhancement is found in theoretical heat transfer coefficient in comparison of dimple and plain plate. Above graph shows that the values of heat transfer coefficient are increases with increasing heat input.

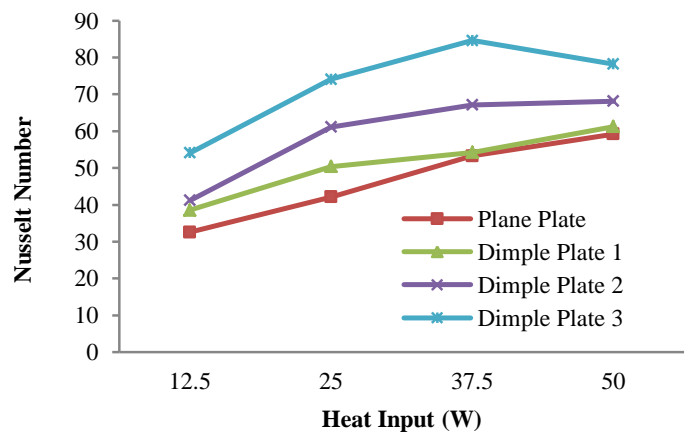

Fig 4.4: Variations in Nusselt Number with Heat Input for various dimple Plates for Aluminum plate.

For increment in heat input, it can be seen from above graphs that enhancement is found in Nusselt Number in comparison of dimple and plain plate. Above figure shows that the values of Nusselt Number are increases with increasing heat input.

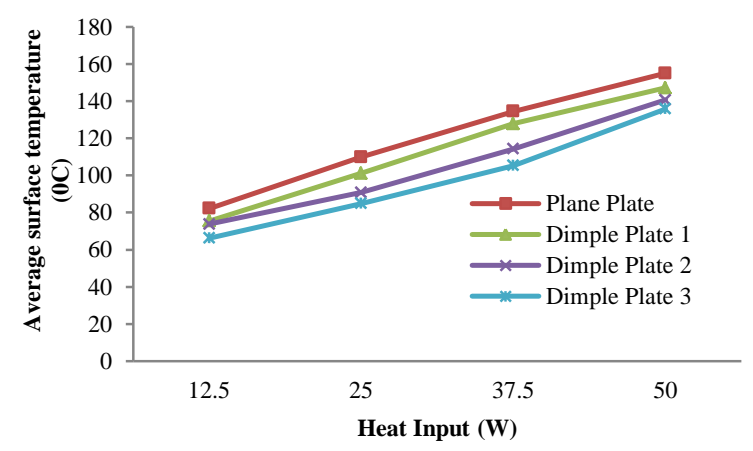

Fig 4.5: Variations in Surface temperature with Heat Input for various dimple plate for Aluminum plate.
For increment in heat input, it can be seen from above graphs that enhancement is found in Surface Temperature in comparison of dimple and plain plate. Above figure shows that the values of Surface temperature are increases with increasing heat input.

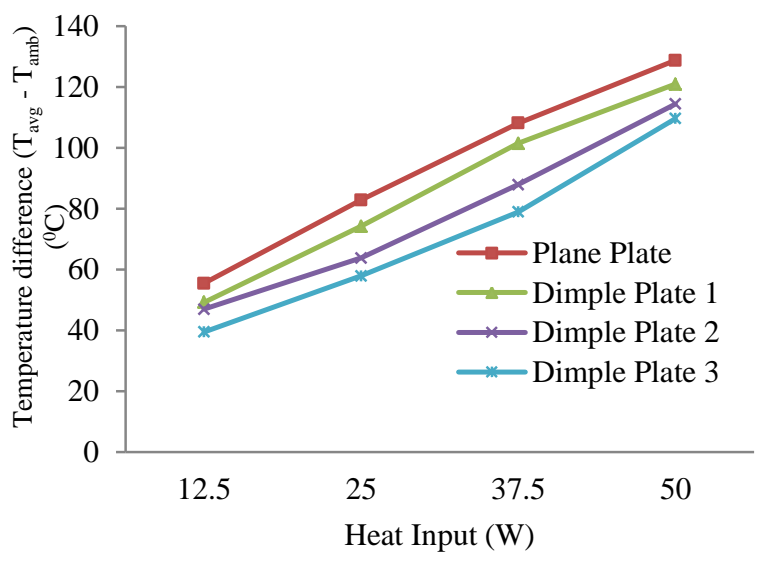

Fig 4.5: Variations in temperature difference with Heat Input for various dimple plate for Aluminum plate.

For increment in heat input, it can be seen from above graphs that enhancement is found in temperature difference in comparison of dimple and plain plate. Above figure shows that the values of Surface temperature are increases with increasing heat input.

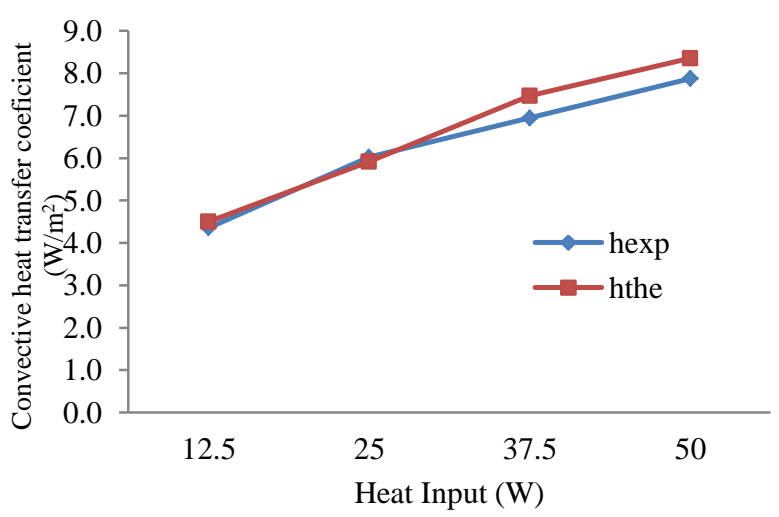

Fig 4.1: Comparison of heat transfer coefficient calculated experimentally and theoretically for plane plate

Above graphs shows that the value of convective heat transfer coefficient calculated by experimental and theoretical method is similar. From this graphs it is proved that experimental setup is validated.

\section{Conclusion}

Experimentation is carried out for the analysis of dimple plates with two different materials (copper and aluminum). Dimple plates are structured with three different dimple patterns for Nusselt number and heat flux in the ranges of 27 to 98 and 3.61 to $1.89 \mathrm{~W} / \mathrm{m}^{2} \mathrm{k}$, respectively. 
It is found that the dimple surface has a significant effect on the enhancement of heat transfer and Nusselt Number. The heat transfer rate is higher as dimple of plate is increases. For a given heat input Nusselt number and heat flux, the average plate temperatures at higher dimple diameter are higher than those from lower dimple diameter. However, heat transfer rate is increases as the heat supplied to plate is increases Therefore, the surface temperature of plate tends to increase as the heat supplied to plate increases. The surface temperature continues to increase with Nusselt number. The measured heat transfer coefficient obtained from the dimple surface at higher diameter is 1.66 times higher than those from the plane surfaces.

\section{References}

Kuethe A. M.,.Boundary Layer Control of Flow Separation and HeatExchange, U.S. Patent No. 3,578,264 1971.

M.A.Dafedar, Mujtabalayeeq I, Mohemmed TaherM, MohammadIdress urf Shahid I Heat Transfer Enhancement Through Different Circular Diametrical Dimple Surface Under Forced Convection -An Experimental Approach, IJRET: International Journal of Research in Engineering and Technology Volume: 02 Issue: 07 | Jul-2013

Iftikarahamad H. Patel, Dr. Sachin L. Borse, Experimental Investigation Of Heat Transfer Enhancement Over The Dimpled Surface, International Journal of Engineering Science and Technology (IJEST), ISSN : 0975-5462 Vol. 4 No.08 August 2012.

Faheem Akthar, Abdul Razak R Kaladgi and Mohammed Samee A Dafedar, Heat Transfer Enhancement Using Dimple Surfaces Under Natural Convection-An Experimental Study, Int. J. Mech. Eng. \& Rob. Res. 2015, ISSN 2278 - 0149 Vol. 4, No. 1, January 2015
Saurabh R Verma P. M. Khanwalkar, V. N. Kapatkar, A Review on Heat Transfer Augmentation for Various Dimpled Geometries, International Journal on Theoretical and Applied Research in Mechanical Engineering (IJTARME), ISSN: 2319-3182, Volume -4, Issue-1, 2015

Amjad Khan, Mohammed Zakir Bellary, Mohammad Ziaullah, Abdul Razak Kaladgi, An Experimental Study on Heat Transfer Enhancement of Flat Plates Using Dimples, American Journal of Electrical Power and Energy Systems, ISSN: 2326-9200, May 28, 2015.

N.K. Burgess and P.M. Ligrani, Effects of dimple depth on channel nusselt numbers and friction factors, J. Heat Transfer 127 (8) (2005) 839-847

G.I. Mahmood and P.M. Ligrani, Heat Transfer in a dimpled channel: combined influences of aspect ratio, temperature ratio, Reynolds number, and flow structure, Int. J. heat Mass Transfer 45 (2002) 2011-2020.

G.I. Mahmood, M.L. Hill, D.L. Nelson, P.M. Ligrani, H.K. Moon and B. Glezer, Local heat transfer and flow structure on and above a dimpled surface in a channel, J. Turbo machinery 123 (2001) 115-123.

S. D. Hwang, H. G. Kwon, H. H. Cho, -Local heat transfer and thermal performance on periodically dimpleprotrusion patterned walls for compact heat exchangers, || Energy, 2010, vol. 35, pp. 5357-5364

Moon, H.K., O Connell, T., and Glezer, B."Channel Height Effect on Heat Transfer and Friction in a Dimpled Passage" J of Eng. For Gas Turbines and Power, Vol.122, pp.307-313, 2000.

Sandeep S. Kore \& Narayan K.Sane, .International Journal of Engineering Science and Technology (IJEST), Vol. 3 , pp 56 - 58, August 2011 